\title{
Direct Visual Internal Urethrotomy in Supine Position in a Patient with Complex Deformities of Both Lower Limbs and Neurogenic Bladder: A Case Report
}

\author{
Balantine U. Eze, Frank K. Chacha, and Timothy U. Mbaeri
}

\section{ABSTRACT}

Direct visual internal urethrotomy (DVIU) is a minimally invasive treatment for urethral stricture and is usually done in lithotomy position. We presented a case of a 35-year-old man with complex deformities of both lower limbs from birth. The lower limbs were severely wasted with ankylosis of the hips, flexion of the knee joints and dorsiflexion at the ankle joints. He had a history of progressively worsening difficult in urination characterized by frequency, urgency, urgency incontinence, nocturia, poor urinary stream (improved by straining), intermittency and feeling of incomplete bladder emptying. He had occasional dysuria and total hematuria. He was not a known hypertensive or diabetic patient. No history of trauma, previous urethral instrumentation, and no history of purulent urethral discharge before the onset of problems. On presentation, his abdomen was full with slight suprapubic distention. The anal sphincter was spastic and the prostate was not enlarged. He had normal noncircumcised male external genitalia. There was no spinal deformity and the upper limbs were normal. White cell count was 14,000 cells/ $\mathrm{mm} 3$ with a differential neutrophil of $85.5 \%$ and urine culture showed moderate growth of coliforms. Abdominopelvic ultrasound showed a thickened bladder wall with mild hydronephrosis bilaterally and a retrograde urethrography and micturating cystourethrography showed 3 short segment bulbar urethral strictures. There was also a Christmas tree appearance of the bladder. A diagnosis of bladder outlet obstruction secondary to multiple short segment idiopathic bulbar urethral strictures on background neurogenic bladder was made. He had intravenous antibiotics for $\mathbf{4 8}$ hours and subsequently a DVIU under spinal anesthesia and in the supine position. Catheter was removed on the 7 th day post procedure and he started clean intermittent catheterization (CIC) with 12 French catheters. Seven months post procedure, patient is still satisfied with the outcome of his treatment. We concluded that DVIU can be done safely in the supine position and CIC can help improve post procedure outcome and in managing comorbid neurogenic bladder.

Keywords: Clean intermittent catheterization, Direct visual internal urethrotomy, Neurogenic bladder, and Supine position.
Published Online: January 30, 2021

ISSN: 2593-8339

DOI: $10.24018 /$ ejmed.2021.3.1.685

B. U. Eze*

Urology Division, Department of Surgery, College of Medicine, Enugu State University of Science and Technology, Enugu, Nigeria.

(e-mail: balantine.eze ${ }^{@}$ esut.edu.ng) F. K. Chacha

Department of Urology, Bugando Medical Centre, Mwanza, Tanzania. (e-mail: frank888990@gmail.com) T. U. Mbaeri

Urology Division, Department of Surgery, Faculty of Medicine, Nnamdi Azikiwe University, Awka, Anambra State, Nigeria.

(e-mail: tu.mbaeri @ ${ }^{\text {unizik.edu.ng) }}$

*Corresponding Author

\section{BACKGROUND}

Direct visual internal urethrotomy (DVIU) is the most frequently performed minimally invasive treatment for urethral strictures in contemporary urology practice [1]. The urethrotomy can be done with either a cold knife or laser. The procedure is typically performed in lithotomy position under spinal or general anesthesia [2]. It can also be done under local anesthesia in selected patients [3].

The success rate of DVIU is variable and depends on disease factors (location of stricture, length of stricture, etiology, degree of spongiofibrosis and preoperative maximum urine flow rate) [2], [4] and patients factors (age and body mass index) [2]. To improve the success rate of DVIU, various adjunctive procedures have been adopted and the most preferred post DVIU adjunctive procedure is clean intermittent catheterization (CIC) [5], [6]. Also, CIC with 12-14 French catheters is the gold standard of management of neurogenic bladder [7] and hence can help improve outcome of management of patients with coexistent neurogenic bladder.

\section{CASE PRESENTAtion}

We present a case of a 35-year-old unmarried man with complex deformity of both lower limbs from birth. The deformity of the lower limbs consisted of severe wasting of the lower limbs with ankylosis of the hips, flexion of the knee joints and dorsiflexion at the ankle joints. He presented with a history of progressively worsening difficult in 
urination. Difficulty in urination consisted of frequency, urgency, urgency incontinence and nocturia. He also had poor urinary stream (that was improved by straining), intermittency and feeling of incomplete bladder emptying. $\mathrm{He}$ had occasional dysuria and total hematuria. With worsening of symptoms, he went to a private hospital (to see a general practitioner) where attempt at urethral catheterization failed before he was referred to us for specialist care. He was not a known hypertensive or diabetic patient. No history of trauma, previous urethral instrumentation and no history of purulent urethral discharge before the onset of problems. On presentation, he was found to be afebrile, not pale, anicteric and not dehydrated. His vital signs were temperature of 37 degrees Celsius, pulse rate 117 beats per minute (full volume and regular), respiratory rate of 21 breaths per minute and blood pressure of $108 / 68 \mathrm{mmHg}$. Abdomen was full with slight suprapubic distention, moves with respiration, no areas of tenderness and he had a palpable bladder $5 \mathrm{~cm}$ above the pubic symphysis. The kidneys were not ballotable and the liver and spleen were not palpably enlarged. He had a spastic anal sphincter and the prostate was not enlarged. He had normal non-circumcised male external genitalia. There was no spinal deformity and the upper limbs had normal power, tone, reflexes and sensation. The lower limbs were deformed (as described above) and spastic. Both pain and fine touch sensations were preserved in the lower limbs. Examination findings in the other systems were essentially normal. Full blood count revealed a hemoglogin of $11.8 \mathrm{~g} / \mathrm{dl}$, platelet count of 103,000 cells/ mm3 and white cell count of 14,000 cells $/ \mathrm{mm}^{3}$ with a differential neutrophil of $85.5 \%$. Urine microscopy, culture and sensitivity showed moderate growth of coliforms sensitive to ceftriaxone, ceftazidime, levofloxacin and nitrofuratoin. Abdominopelvic ultrasound showed a thickened bladder wall (6 millimeters) with mild hydronephrosis bilaterally. We did a retrograde urethrography and micturating cystourethrography that revealed two short segment strictures at the distal bulbous urethra and one at the proximal bulbous urethra (Fig. 1: areas of stricture labeled a, b and c). There was also a Christmas tree appearance of the bladder. The serum electrolytes, urea and creatinine were essentially normal. We made a diagnosis of bladder outlet obstruction secondary to multiple short segment idiopathic bulbar urethral strictures on background neurogenic bladder. $\mathrm{He}$ had intravenous antibiotics for 48 hours and subsequently direct visual internal urethrotomy (DVIU). Procedure was done under spinal anesthesia (anesthesia was administered with the patient lying lateral) and urethrotomy done in the supine position (Fig. 2). DVIU with a 21 French DVIU sheath and 0 degrees telescope was performed (for the three strictures) using a cold knife and a 0.035 inch guide wire at 12 O'clock position (Fig. 3).

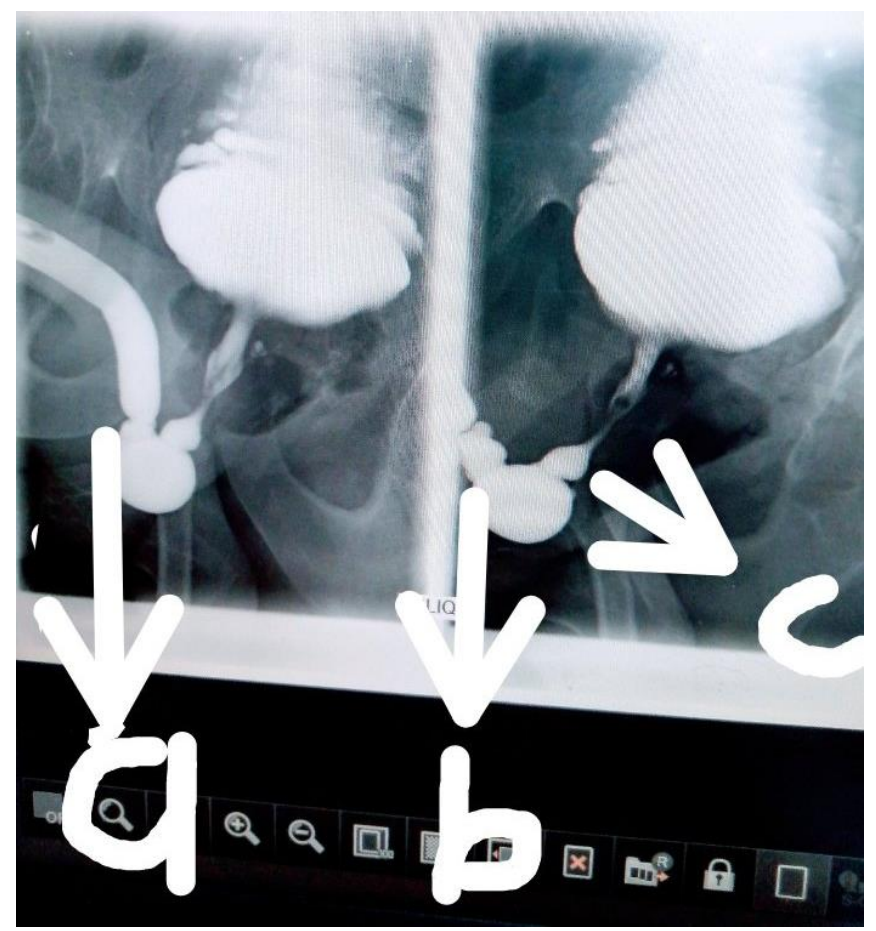

Fig. 1. Retrograde + Micturating Cystourethrogram (a, b, and c marks the stricture points confirmed during endoscopy).

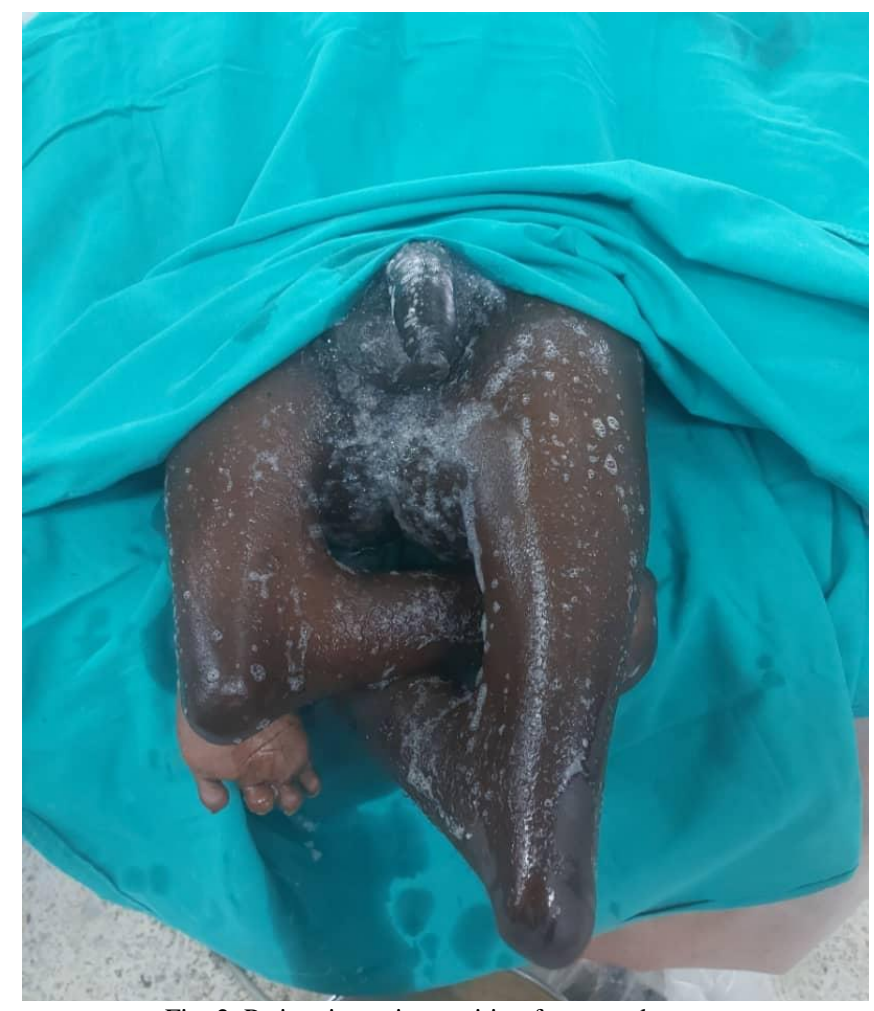

Fig. 2. Patient in supine position for procedure.

Intraoperative findings included 3 short segment bulbar urethral strictures, normal non-obstructing prostate, cloudy urine (Fig. 4) and bladder trabeculations (Fig. 5). Post procedure, a size 16 French catheter was easily passed (Fig. 6). He had intravenous antibiotics for more 48 hours before being discharged home on the 3rd day post procedure on oral antibiotics. Catheter was removed on the 7 th day post procedure. He was taught to do CIC with 12 French catheters at least three times a day to augment bladder emptying. Seven months post procedure, patient is still satisfied with the outcome of his treatment. 


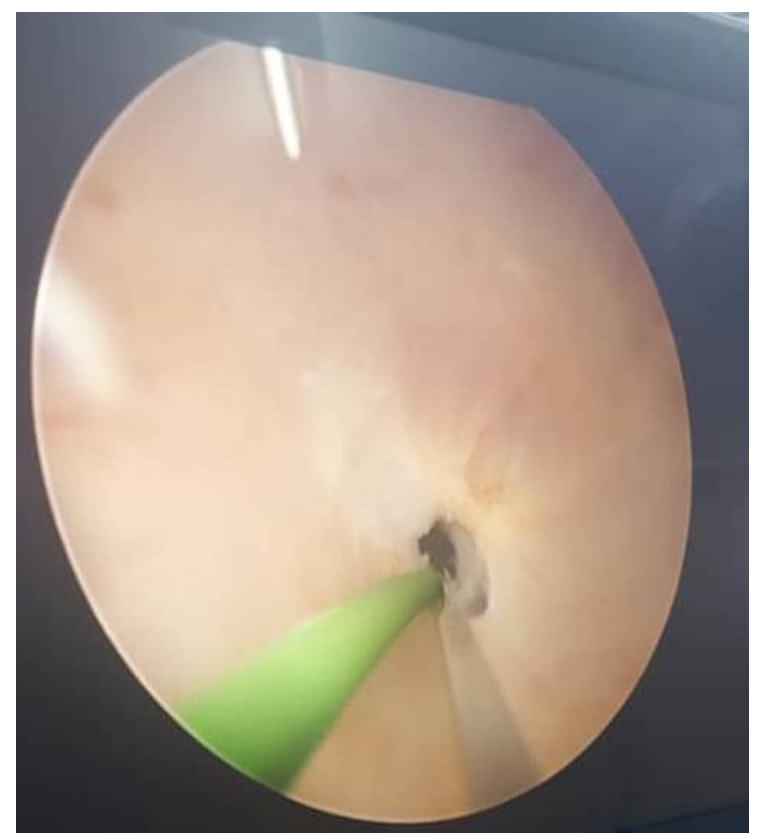

Fig. 3. Urethral stricture and direct visual internal urethrotomy procedure.

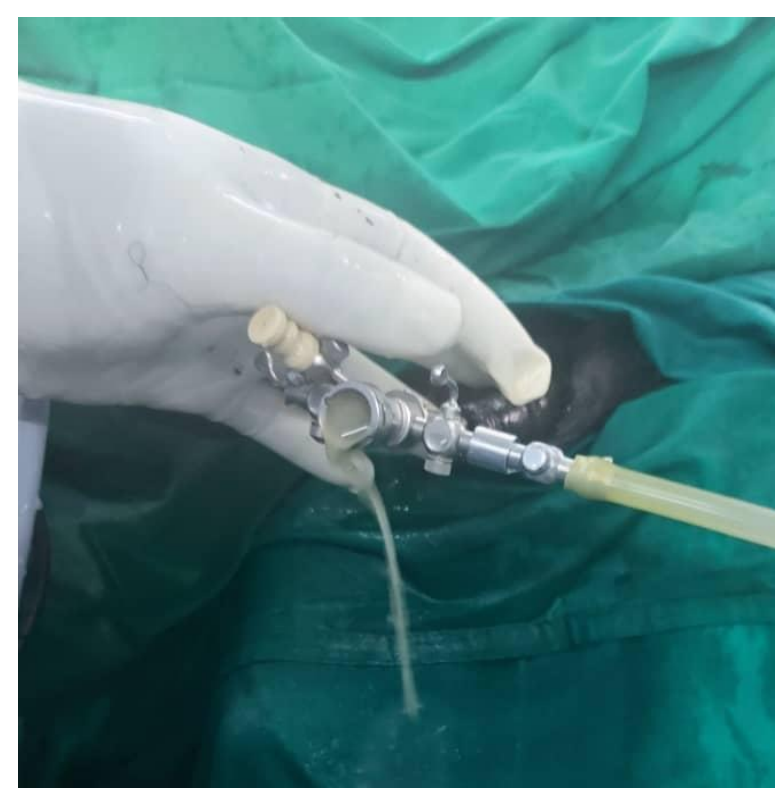

Fig. 4. Egress of cloudy urine.

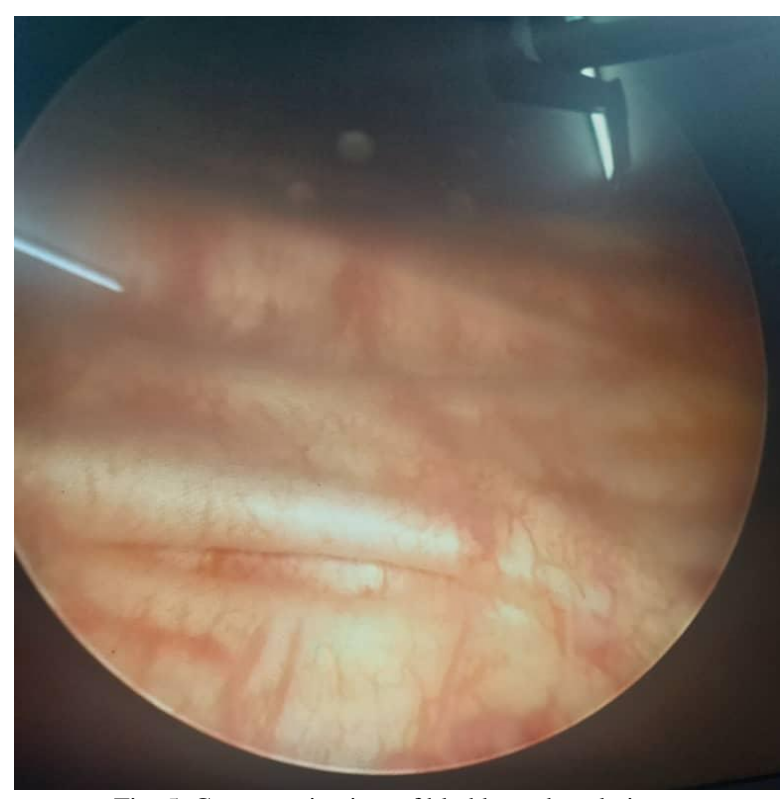

Fig. 5. Cystoscopic view of bladder trabeculations.

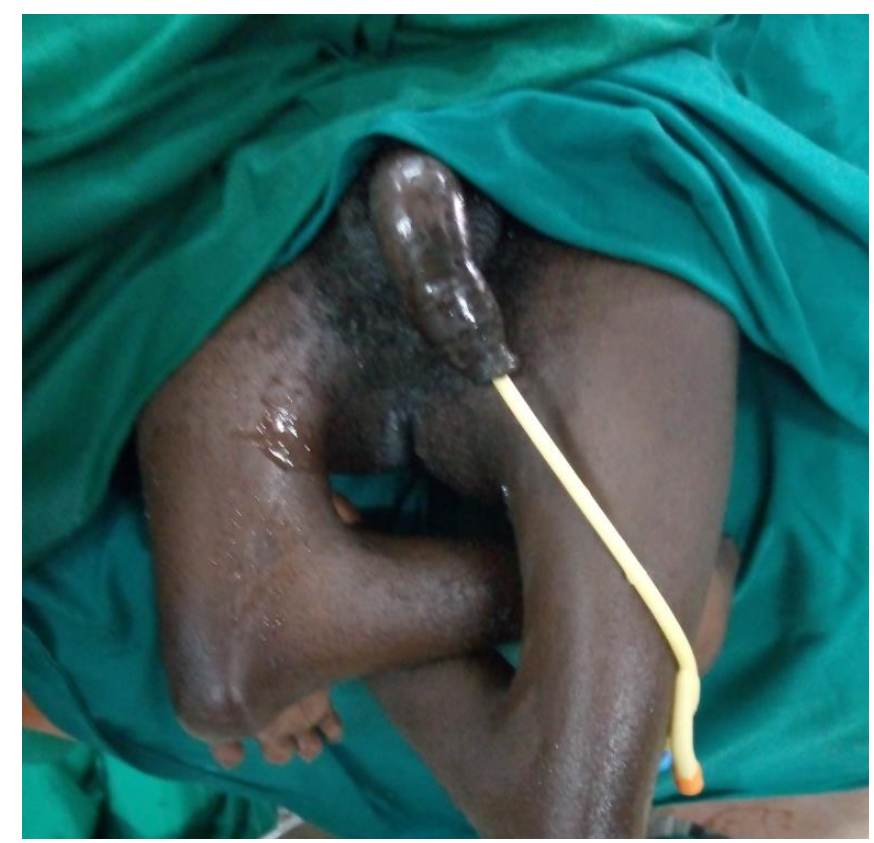

Fig. 6. Post procedure.

\section{Literature REVIEW AND CASE DisCUSSION}

DVIU is a safe, minimally invasive, easy and time effective treatment for initial management of short segment urethral strictures [8], [9] and also has the advantage of a short convalescence period [9]. The position for transurethral management of urethral stricture is usually dorsal lithotomy position with the buttocks of the patient moved to the edge of the bed [10]. There is no significant difference between recurrence rates and complication rates of use of cold knife and Holmium: YAG laser in internal urethrotomy [11]. This suggests that both forms of internal urethrotomy are safe and are effective treatment modalities for selected bulbar urethral strictures.

We had the challenge of having a patient who could not be placed in the dorsal lithotomy position conventionally used for DVIU because of his complex limbs deformities. We were considering a supbrapubic cystotomy (SPC) but on discussion with the patient about options of treatment, he preferred an endoscopic procedure because of the obvious advantages. We also got consent for possible SPC if we failed with the attempt at endoscopic treatment. To the best of our knowledge, there has not been any report in literature about performing DVIU in a supine position. Yeşil et al [12] had to move their patient from the supine to the lithotomy position to enable them to do urethrocystoscopy and DVIU following inability to pass urethral catheter post hypospadias surgery. With this report of successful DVIU in supine position, one wonders if it would have been possible to do the DVIU in the supine position without the inconvenience of repositioning the patient and still achieve a good outcome.

Passage of urethral catheter post DVIU can be difficult sometimes [12]. We did not experience any difficulty in passing urethral catheter post DVIU in our patient despite the limitation of the position he was placed in because of the lower limbs deformities. The urethral catheter was removed $7^{\text {th }}$ day post procedure as research have shown that most urologist leave urethral catheter for around one week 
following DVIU [13].

Our succeeding in doing DVIU has enabled our patient to commence clean intermittent catheterization (CIC). CIC following DVIU reduces the rate of recurrence of urethral stricture and improves quality of life of patients [5]. For our patient, the CIC has the dual role of improving the outcome of the DVIU and ameliorating the coexistent problem of neurogenic bladder.

We are not surprised our patient has done well and is happy as the strictures he had were short segments $(<1$ centimeter) and idiopathic. Following DVIU, idiopathic strictures shave been shown to have more favorable prognosis than iatrogenic strictures [14], [15]. Our challenges still remain what to do in case the strictures recur in the distant or near future. Do we do another DVIU or do we do an open urethroplasty? Open urethroplasty will be very difficult because of difficulty in positioning the patient in a way to gain adequate access to the bulbar urethra. Our patient have refused proper evaluation for the cause of his lower limbs deformities partly due to financial constraint and his reason that he has lived with the condition all his life and knowing the cause is not equivalent to providing cure for the deformities.

\section{CONCLUSION}

Direct visual internal urethrotomy is a viable treatment option for short segment bulbar urethral strictures and can be done safely in the supine position in some selected patients who cannot be placed in the lithotomy position. Post procedure clean intermittent catheterization can help improve the outcome of DVIU and manage concomitant neurogenic bladder.

\section{ACKNOWLEDGMENT}

The authors would like to thank Doctor Innocent J. Bangirana for helping retrieve the medical records of the patient used in this case report.

\section{REFERENCES}

[1] D. Dubey, "The current role of direct vision internal urethrotomy and self-catheterization for anterior urethral strictures," Indian J Urol, vol. 27, no. 3, pp. 392-396, Jul. 2011.

[2] A. M. Harraz, A. El-Assmy, O. Mahmoud, A. A. Elbakry, M. Tharwat, H. Omar, H. Farg H, M. Laymon, and A. Mosbah, "Is there a way to predict failure after direct vision internal urethrotomy for single and short bulbar urethral strictures?," Arab J Urol, vol. 13, no. 4, pp. 277-281, Dec. 2015.

[3] D. Darlington, "A Novel Method of Local Anesthesia for Optical Internal Urethrotomy," Cureus, vol. 11, no. 10, pp. e6043-e6043, Oct. 2019.

[4] G. Barbagli, N. Fossati, F. Montorsi, S. Balò, C. Rimondi, A. Larcher, S. Sansalone, D. Butnaru, and M. Lazzeri, "Focus on internal urethrotomy as primary treatment for untreated bulbar urethral strictures: results from a multivariable analysis," Eur Urol Focus, vol. 6, no. 1, pp. 164-169, Jan. 2020.

[5] A. Jhanwar, A. K. Sokhal, K. Singh, S. Sankhwar, and D. K. Saini, "Assessment of quality of life in patients of urethral stricture on clean intermittent catheterization following direct vision internal urethrotomy," Urol Ann, vol. 10, no. 4, pp. 395-399, Oct. 2018.

[6] V. Kumar, S. Dharwadkar, C. Doshi, and A. Batta, "Role of intermittent self catheterization in prevention of recurrence of stricture urethra following visual internal urethrotomy: a prospective single centre study," Int J Surg, vol. 6, no. 4, pp. 1093-1096, Mar. 2019.

[7] L. Liao, "Evaluation and management of neurogenic bladder: what is new in China?," Int J Mol Sci, vol. 16, no. 8, pp. 18580-18600, Aug. 2015.

[8] K. Sureshkumar, P. K. Shukla, A. P. Gaharwar, M. Jeswani, and S. Sahu, "Role of visual internal urethrotomy in the management of short segment urethral stricture in male," Int J Surg Sci, vol. 4, no.1 pp. 74-77, 2020.

[9] A. G. Cavalcanti, and G. Fiedler, "Opinion: endoscopic urethrotomy," Int Braz J Urol, vol. 41, no. 4, pp. 619-622. Aug. 2015.

[10] J. Furr, and J. Gelman, "Endoscopic Management of Urethral Stricture Disease and Bladder Neck Contractures," J Endourol, vol. 34, no. S1, pp. 7-12. May 2020.

[11] M. G. Yenice, K. G. Seker, E. Sam, Y. Colakoglu, F. A. Atar, S. Sahin, A. Simsek, and V. Tugcu, "Comparison of cold-knife optical internal urethrotomy and holmium: YAG laser internal urethrotomy in bulbar urethral strictures," Cent European J Urol, vol. 71, no. 1, pp. 114-120, 2018.

[12] S. Yeşil, A. Atan, and F. Polat, "A method that facilitates urethral catheterization after internal urethrotomy," Turk J Urol, vol. 45, no. S1, pp. S125-S127, Dec. 2019.

[13] G. G. Ferguson, T. L. Bullock, R. E. Anderson, R. E. Blalock, and S. B. Brandes, "Minimally invasive methods for bulbar urethral strictures: a survey of members of the American Urological Association," Urology, vol. 78, no. 3, pp. 701-706, Sept. 2011.

[14] P. Albers, J. Fichtner, P. Bruhl, and S. C. Muller, "Long-term results of internal urethrotomy," J Urol, vol. 156, no. 5, pp. 1611-1614, Nov. 1996.

[15] A. Chandra, and S. K. Singh, "Internal Urethrotomy for Anterior Urethral Strictures, its Place in the Current Management of Stricture Disease and Role of CSIC in Prevention of Recurrence," JAMDSR, vol. 7 , no. 11 , pp. 168-170, Nov. 2019. 